\title{
Novel Joint Angle Measurement and Calibration System for Selective Compliance Assembly Robot Arm
}

\author{
Chun-Kai Lan, Chung-Yu Wu, Chih-Wen Chang, \\ Hau-Wei Lee, and Chien-Hung Liu* \\ National Chung Hsing University, No. 145 Xingda Road, South District, Taichung City, Taiwan
}

(Received March 28, 2018; accepted August 31, 2018)

Keywords: SCARA robotics, position-sensitive detector, robotic end effector

In this study, a laser measurement device was designed to measure the axial angle errors of a selective compliance assembly robot arm (SCARA) joint. The proposed measurement system includes an optical measurement device that is installed on a high-precision index table and a reflective mirror that is installed on the end effector of the SCARA. The optical measurement device consists of a one-dimensional position-sensing detector, a polarized beam splitter (PBS), a laser diode (LD), a 1/4-wave plate ( $\lambda / 4 \mathrm{WP})$, a collimating lens $(\mathrm{C})$, and a reflective mirror. The device is easy to operate, inexpensive, and highly accurate. The axial angle error of the SCARA joint can be measured using the measurement device and a high-precision index table after calibration. The experimental results show that the positioning accuracy of the J1 axis improved from 25.6 to 8.1 arcsec, and that of the J2 axis improved from 7.3 to 2.3 arcsec after the angular positioning error was compensated.

\section{Introduction}

Robotic arms have been widely used for industrial automation, and industries in different countries around the world need robotic arms for different applications. Robotic arms have repeatable accuracy, and are designed to replace manpower in the execution of repeated commands or dangerous work. The use of multijoint robotic arms is particularly widespread among car manufacturers, auto parts makers, and electronics industries. The technology and quality of production are enhanced by using robotic arms, which can carry out most of the tasks at the initial stage of production. With accuracy, precision, and zero error, robotic arms are advantageous in assuring the quality of production. The operating or processing accuracy is a factor that must be considered with respect to all the tasks during the process of production, including pick-and-place, welding, and assembly. The operating accuracy of robotic arms is determined by the positioning error or deviation of the relative motion between the arm and the workpiece. Most factories rely on robotic arms that operate through multijoint rotation to enable $2 \mathrm{D}$ or $3 \mathrm{D}$ motion. Hence, the key question is whether the joint of a robotic arm can rotate to an accurate position during the motion of the arm. The selective compliance assembly

*Corresponding author: e-mail: carus@dragon.nchu.edu.tw https://doi.org/10.18494/SAM.2018.2080 
robot arm (SCARA) has a wide range of motion and moves fast. It has a simple structure, is reasonable in cost, and is ideal for fast pick-and-place or assembly applications. In this study, a SCARA is selected as the model for the development and design of measurement systems, programming, and calibration to meet the requirements of industries. The required positions and directions are calculated using the given vertex angle of the end effector. The errors of the position and direction are important performance indicators of a robotic arm. The past years saw a significant increase in the need for path accuracy, and various calibration methods were developed in the past decade. For example, the inverse kinematics robot calibration is accomplished by measuring errors within the entire working range of an end effector at a discrete point, whereas in the high-throughput target motion calibration method, the deviation of the nominal parameter is measured in the geometrical model of the robot. On the other hand, the linear displacement sensor, theodolite, 3D laser tracker, and CCD image capture method are used to calibrate the accuracy of the robotic arm. ${ }^{(1)}$

Giovani et al. ${ }^{(2)}$ predicted the errors of a robotic path by a laser optical measurement method designed for the static calibration of industrial robots. The result showed that a slight movement of the joint could increase the accuracy of the robotic arm. Alberto et al. ${ }^{(3)}$ compared three methods for the identification of geometrical parameters to improve the kinetic accuracy of a SCARA. They measured the path error at the end of the robotic arm using laser triangulation measurement technology, and the result confirmed the applicability of the method. Liu et al. ${ }^{(4)}$ proposed a joint displacement calibration method for industrial robots. A device installed on the end of a robotic arm shoots laser beams from different positions and in directions toward the surface of the position-sensitive detector (PSD), and the feedback from the PSD was used for positioning. Ideally, the intersection of each pair of laser beams converges on the same point after displacements are compensated. Gatla et al. ${ }^{(5)}$ described the method for a virtual closed kinematic chain. Two fixed points are randomly chosen to create a laser measuring tool for the end-point effects of a virtual closed kinematic chain. The result showed that the kinematic parameters of robots must be calibrated.

Biqiang et al. ${ }^{(6)}$ installed a laser shooting device on the end effector of a robot. The device aims at PSD components and shoots focused laser beams toward the two centers of the PSD, creating a virtual linear constraint on the end effector of the robotic arm. The highly precise feedback from the PSD increases the resolution of the observed values and improves the measurement accuracy of the robot's joint angle. By the closed-loop measurement method, the robotic arm can be calibrated by using the sensor installed inside the robotic arm and the kinematic constraints of the mechanism. ${ }^{(7,8)}$ Osborn and Newman ${ }^{(9)}$ designed a measurement system with a four-quadrant PSD installed at the end point of a robotic arm. The measurement is made by moving the robotic arm along a laser line to a linear displacement and using the four-quadrant PSD to measure the horizontal and vertical errors of the center of the laser beam when the end effector of the robotic arm moves to each of the sampling points. Zhuang et al. ${ }^{(10)}$ designed a measurement system on the assumption that a robotic arm can move across three datum planes and check for accurate contact distance between the tool nose and the planes, while collecting the readings of the joint position sensors equipped inside the robotic. The number of measurement points must be more than the number of the parameters calibrated in 
the whole system. Yin and $\mathrm{Gao}^{(11)}$ studied a five-degree-of-freedom (DOF) articulated robotic arm. A laser tracker was used as the calibration device, and a reflective mirror with a motor rotation axis was installed on the flange face at the end of a robotic arm. The laser beams emanating from the laser tracker tracked the parallel position of the reflective mirror. In this way, the pitch and yaw angles of joint axes can be acquired. Measurement software is used to identify the position of every point in the reference coordinate system and calibrate the DenavitHartenberg parameters (DH parameters). Tong et al. ${ }^{(12)}$ used a laser checker to measure the repeatable positioning accuracy of a SCARA at different feed rates in different positions. The result showed that a proper moving speed can improve the repeatable accuracy in a continuous path.

\section{Principles of Measurement}

\subsection{SCARA}

The SCARA (Syntec THL 600 series) is a two-DOF planar articulated robotic arm with a ball screw that enables movement toward any position within the $X Y$ plane. The movement of the robotic arm can be inferred from direct kinematics using the following equations to analogically compute the position of the end. The joint axes J1, J2, and R of the SCARA are rotational, while the $z$-axis can move linearly. The working range looks like a cylindrical sector when the four axes are in motion. The following equations can be used to calculate the $x$ and $y$ positions of the SCARA robotic end effector.

$$
\begin{aligned}
& x=L_{1} \cos \theta_{1}+L_{2} \cos \left(\theta_{1}+\theta_{2}\right) \\
& y=L_{1} \sin \theta_{1}+L_{2} \sin \left(\theta_{1}+\theta_{2}\right)
\end{aligned}
$$

Here, $\theta_{1}$ is the angle of rotation of the $\mathrm{J} 1$ axis, $\theta_{2}$ is the angle of rotation of the $\mathrm{J} 2$ axis, $L_{1}$ is the length between $\mathrm{J} 1$ and $\mathbf{J} 2$, and $L_{2}$ is the length between $\mathrm{J} 2$ and $\mathrm{R}$. Thus, the angles $\theta_{1}$ and $\theta_{2}$ can be determined by the inverse kinematics of the robotic arm, as in the following equations:

$$
\begin{gathered}
\theta_{1}=\tan ^{-1} 2(X, Y)-\cos ^{-1}\left(\frac{x^{2}+y^{2}+L_{1}^{2}-L_{2}^{2}}{2 L_{1} \sqrt{x^{2}+y^{2}}}\right), \\
\theta_{2}=\cos ^{-1}\left(\frac{x^{2}+y^{2}+L_{1}^{2}-L_{2}^{2}}{2 L_{1} L_{2}}\right) .
\end{gathered}
$$

Table 1 shows the specifications of the SCARA robotic arm used in this study. 
Table 1

Specifications of robotic arm.

\begin{tabular}{lc}
\hline Linkage length from J1 to J2 & $300 \mathrm{~mm}$ \\
Linkage length from J2 to R & $300 \mathrm{~mm}$ \\
Max. radius of rotation & $600 \mathrm{~mm}$ \\
$Z$-axis traveling range & $400 \mathrm{~mm}$ \\
$R$-axis angle of rotation & $180^{\circ}$ \\
Max. load & $10 \mathrm{~kg}$ \\
Repeatability & $\pm 0.025 \mathrm{~mm}$ \\
\hline
\end{tabular}

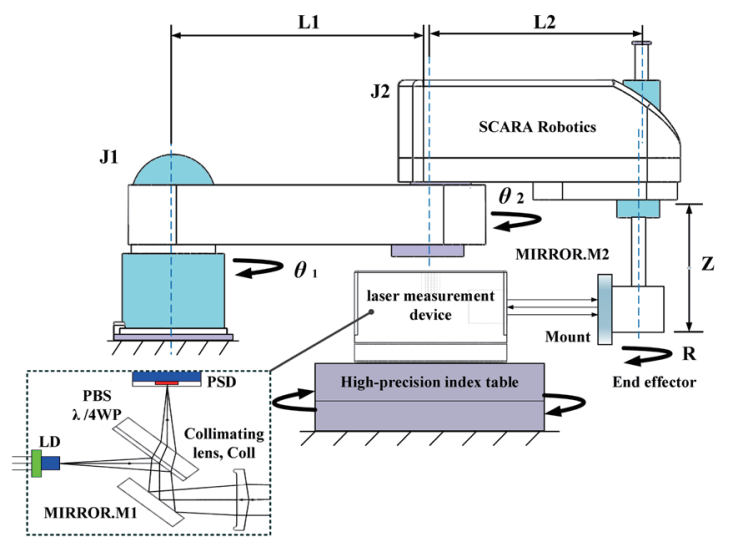

Fig. 1. (Color online) Setup of the measurement system.

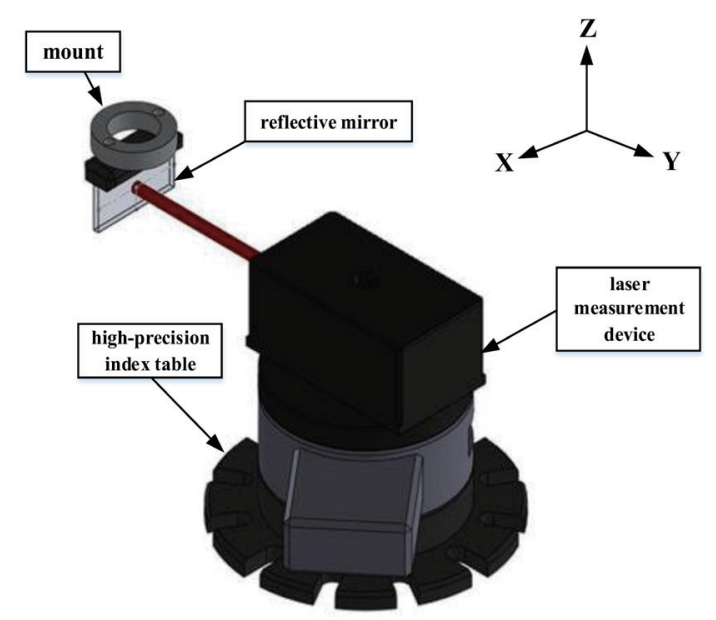

Fig. 2. (Color online) Components of the measurement system.

\subsection{Structure of the measurement system}

The proposed measurement system is developed for measuring the angular positioning error of the SCARA. The measurement structure is shown in Fig. 1. The measurement system includes a high-precision index table, a small-angle measurement device, and a reflective mirror, as shown in Fig. 2. The small-angle measurement device consists of a laser diode (LD), a polarized beam splitter (PBS), a $\lambda / 4$-wave plate $(\lambda / 4 \mathrm{WP})$, a collimating lens $(\mathrm{C})$, a reflective mirror, and an 1D PSD.

The light path is as shown in Fig. 1. The laser beam is emitted from the LD through the $\lambda / 4 \mathrm{WP}$ and PBS to form a parallel polarized beam. It is then reflected off the first reflective mirror (M1), travels through the $\mathrm{C}$, and is reflected off the second reflective mirror (M2) back to the original path. The light again passes through the $\lambda / 4 \mathrm{WP}$ to form a vertical polarized beam that enters the PBS and is projected onto the 1D PSD. If M2 is moved by a small angle, the measurement device can measure this small angle in accordance with the autocollimator principle. ${ }^{(13-15)}$ M2 is installed on the end effector through a mount. The information on the rotation angle errors of the SCARA axis is transmitted back through M2. Since the measurable range of the measurement device is within \pm 200 arcsec, a high-precision index table is used for wide-range measurement (e.g., larger than $\pm 90^{\circ}$ ). The index table can be controlled by a computer. The angular positioning repeatability and accuracy of the index table are 0.2 and 1.0 arcsec, respectively. 


\subsection{Measurement method}

The angular positioning error measurement method is as shown in Fig. 3. The basis of the above-mentioned method of the proposed measurement system is polygon angle calibration. ${ }^{(16)}$ The differences are that, for the polygon calibration system, a high-precision index table is placed on a table, and a polygon is placed on the index table. The commonly used small-angle measurement device for polygon angle calibration is an autocollimator, which is placed in front of the polygon. ${ }^{(17)}$ The rotating angle amount for one step of the index table equals the angle deviation of the polygon. Thus, the small-angle deviation of the polygon can be measured using the autocollimator.

For the proposed measurement system, a reflective mirror (M2) is installed on the end effector of the SCARA. The maximum distance between this reflective mirror and the measurement device is $300 \mathrm{~mm}$. The small-angle measurement device is placed on the high-precision index table. The SCARA and the index table are rotated at the same angle simultaneously. Thus, the small-angle deviation of the SCARA is measured. For instance, let the angle of the J1 axis of the SCARA be $\theta_{1}$ and the angle of the index table be $\theta_{t}$; the relationship between $\theta_{1}$ and $\theta_{t}$ is

$$
\theta_{1}=\theta_{t}+\delta_{\theta 1}
$$

where $\delta_{\theta}$ is the small-angle deviation, which is measured using the small-angle measurement device. Similarly, the small-angle deviation of the J2 axis can be computed as

$$
\theta_{2}=\theta_{t}+\delta_{\theta 2}
$$

From Eqs. (5) and (6), the determined small-angle deviation can be used for angular positioning error compensation for the $\mathrm{J} 1$ and $\mathrm{J} 2$ axes.

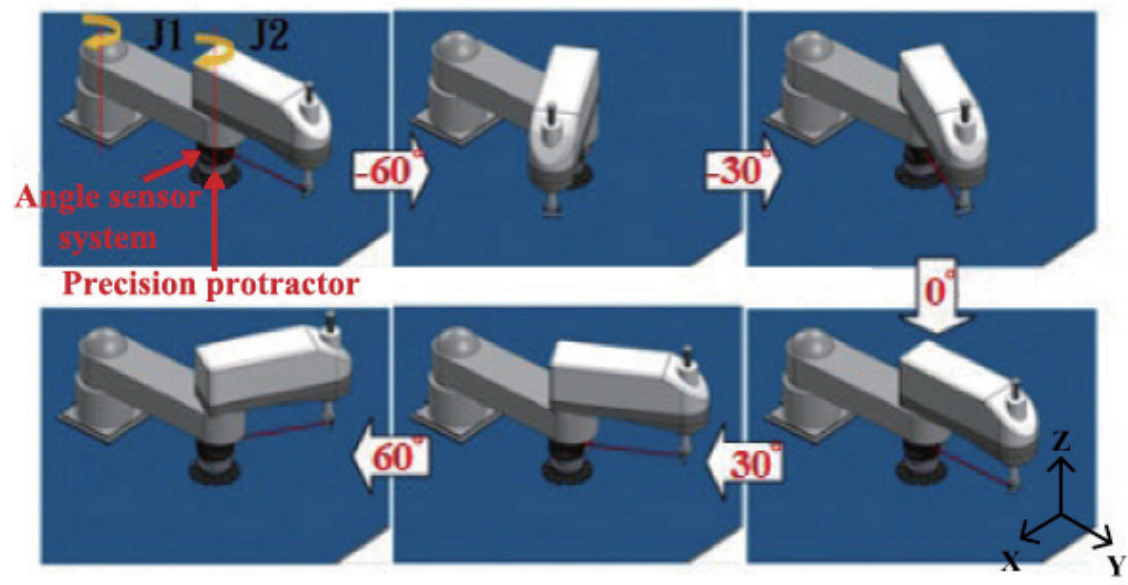

Fig. 3. (Color online) Steps of angular positioning error measurement method. 


\subsection{Calibration of the measurement system}

After the measurement system was designed and assembled, it was calibrated with a highprecision measurement device to ensure accuracy. The system was also tested three times to ensure stability. In this study, calibration and verification of the measurement system were conducted using the rotational precision (RP) checker designed in our lab. The displacement $\Delta \theta$ was calibrated in the experiment. The reflective mirror (M1) was placed in the RP checker to generate a slight displacement. Calibration was repeated three times to maintain the repeatable accuracy of the system. Figure 4 shows the linear curve of the calibration of the system and Fig. 5 shows the residual error. With respect to the stability of the system, the standard deviation voltage was substituted into the linear curve equation, and converted to the displacement, as shown in Fig. 6. The specifications of the SCARA robotic angle sensor are as shown in Table 2.

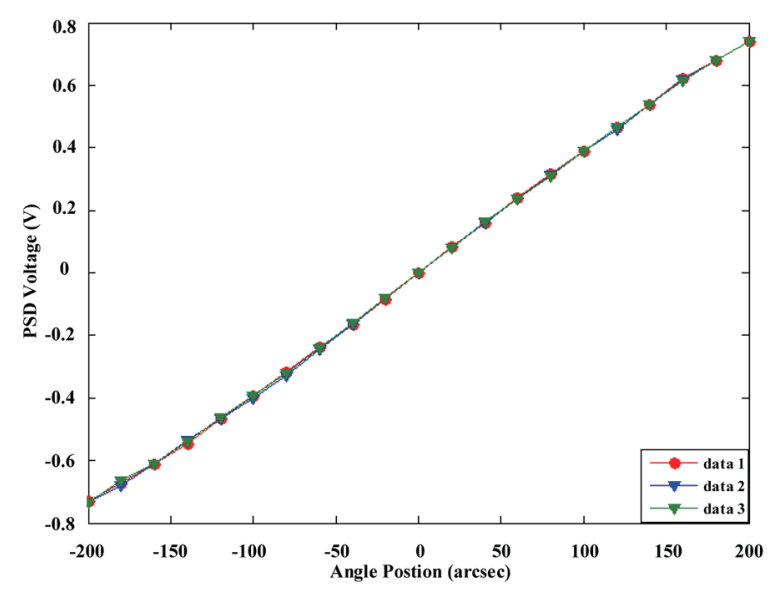

Fig. 4. (Color online) Linear curve.

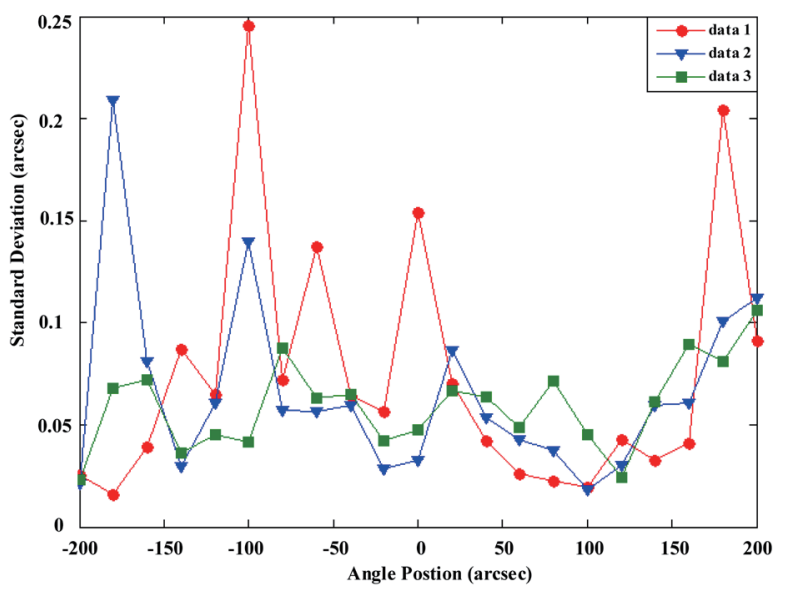

Fig. 6. (Color online) Standard deviation.

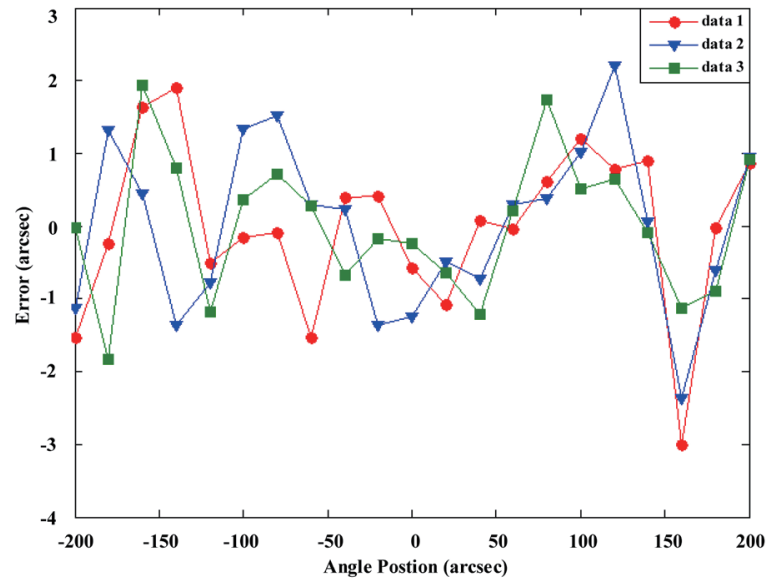

Fig. 5. (Color online) Error of residual.

Table 2

Specifications of the SCARA robotic angle sensor.

Resolution 0.5 arcsec

Repeatability $\quad 1$ arcsec

Accuracy $\pm 3 \operatorname{arcsec}$

Measurable range of the small- $\quad$ within $\pm 0.1^{\circ}$ angle measurement device

Traveling range of the high-

5 to $360^{\circ}$ 


\section{Verification and Experimental Results}

In the measurement system in this study, we adopted the signal acquisition card (NI USB6210) to send and receive signals. The sampling rate of the signal acquisition card is $10 \mathrm{kHz}$. LabVIEW was used for the programming of the measurement system. The measurement system developed in this study focused on the joint angle error measurement of the SCARA robotic arm of the J1 and J2 axes, and the calibration results were analyzed in accordance with the VDI 3441 standards. Then, we used a laser interferometer to carry out the $y$-axis data analysis verification of linear length measurement before and after the compensation for the joint axes J1 and J2. Figure 7 shows the setup of the experiments.

\subsection{Robotic arm verification results}

Figure 8 shows the experimental result of the joint axis $\mathrm{J} 1$ before calibration. The reversal error is 10.4 , the positioning accuracy is 151.7 arcsec, the repeatable precision is 9.7 arcsec, and the position deviation is 140.8 arcsec. Figure 9 shows the experimental result of the joint axis $\mathrm{J} 1$ after calibration. The reversal error is 8.8 , the positioning accuracy is 26.5 arcsec, the repeatable precision is $8.1 \mathrm{~s}$, and the position deviation is 19.2 arcsec.

Figure 10 shows the experimental result of the joint axis $\mathrm{J} 2$ before calibration. The reversal error is 2.3 , the positioning accuracy is $71.2 \mathrm{arcsec}$, the repeatable precision is $3.2 \mathrm{arcsec}$, and the position deviation is 67.9 arcsec. Figure 11 shows the experimental result of the joint axis J2 after calibration. The reversal error is 2.3 , the positioning accuracy is 7.3 arcsec, the repeatable precision is $2.3 \mathrm{arcsec}$, and the position deviation is $5.2 \mathrm{arcsec}$.

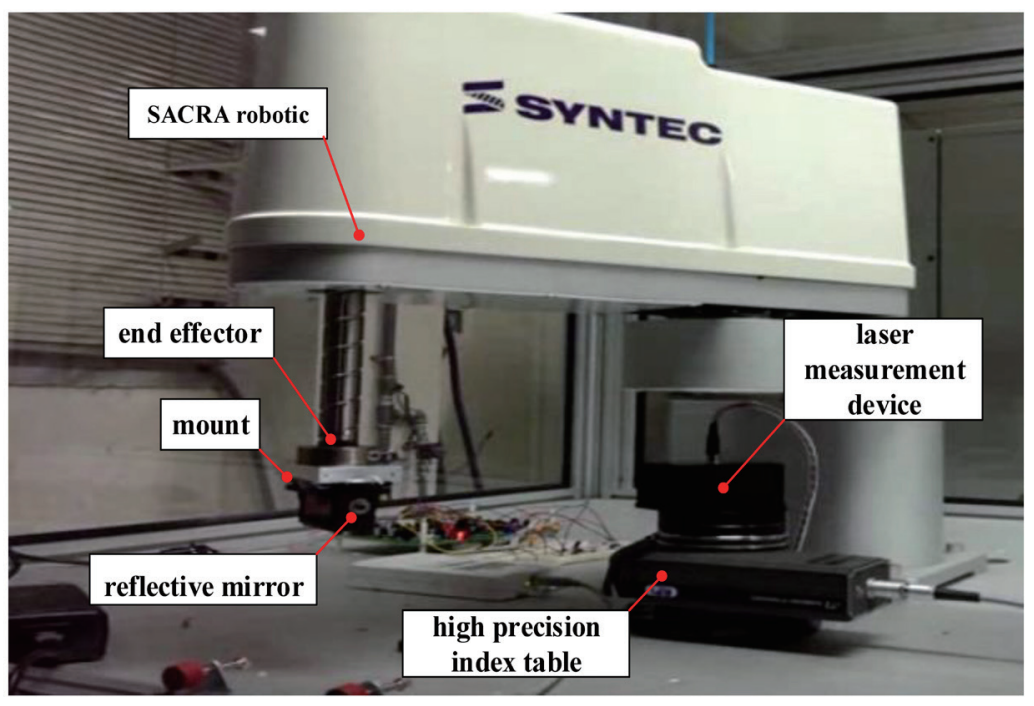

Fig. 7. (Color online) Setup of the experiments. 


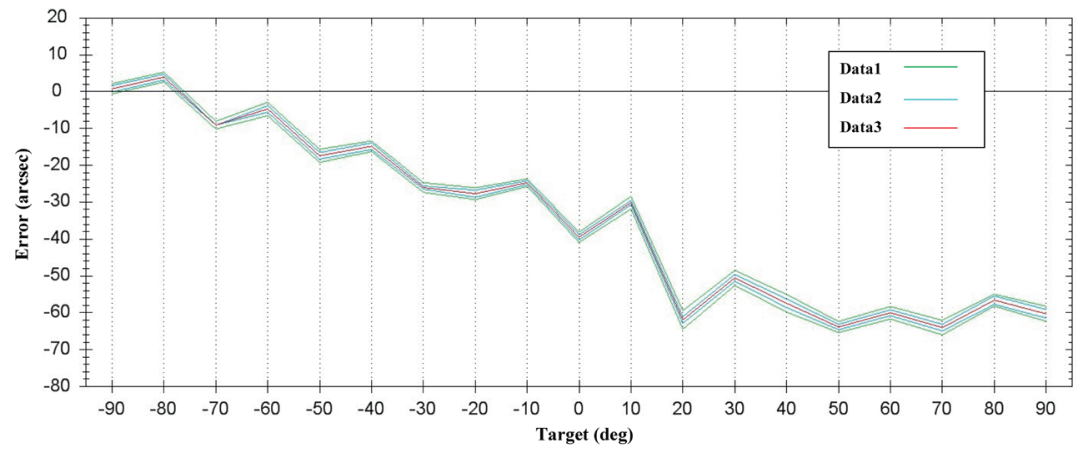

Fig. 8. (Color online) Joint axis J1 before calibration.

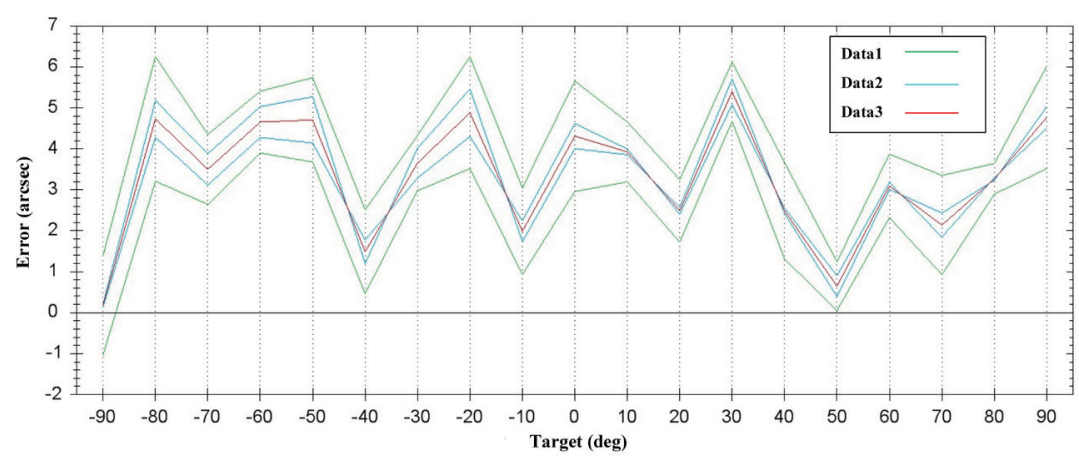

Fig. 9. (Color online) Joint axis J1 after calibration.

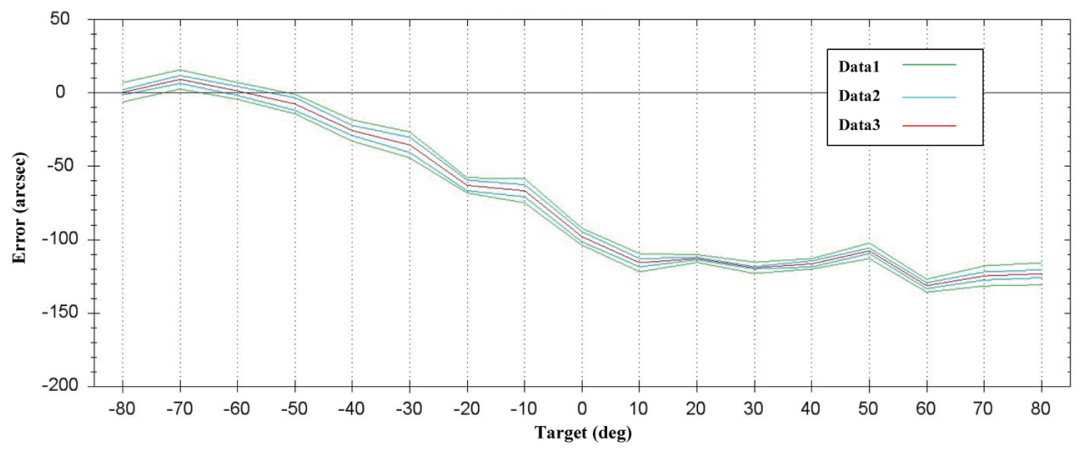

Fig. 10. (Color online) Joint axis J2 before calibration.

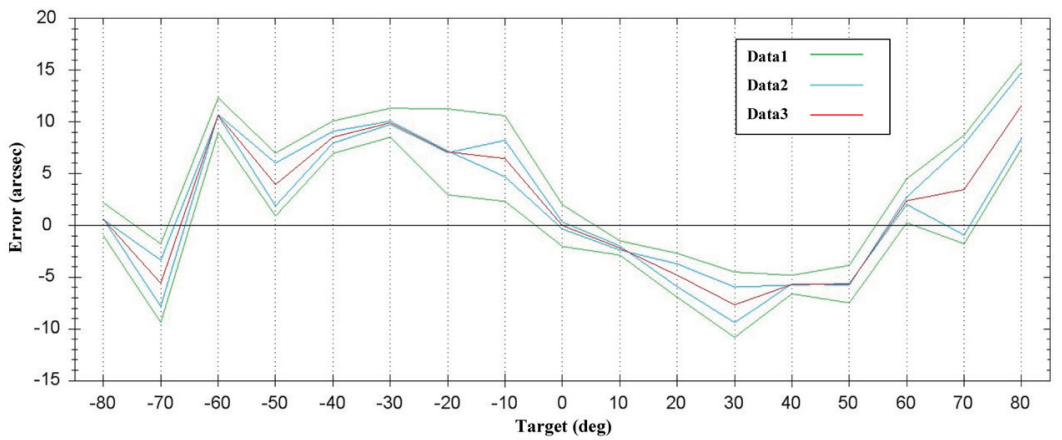

Fig. 11. (Color online) Joint axis J2 after calibration. 


\subsection{Analytical comparison of $y$-axis linear length measurement before and after calibration}

In this study, we used RENISHAW's ML-10 laser interferometer to verify the $y$-axis linear length measurement of the SCARA. The initial measurement is shown in Fig. 12. The reversal error is $90.33 \mu \mathrm{m}$, the positioning accuracy is $1210.69 \mu \mathrm{m}$, the repeatable precision is $12.42 \mu \mathrm{m}$, and the position deviation is $1153.76 \mu \mathrm{m}$. The $y$-axis linear length measurement of the joint axes $\mathrm{J} 1$ and $\mathrm{J} 2$ after calibration is shown in Fig. 13. The reversal error is $55.86 \mu \mathrm{m}$, the positioning accuracy is $578.61 \mu \mathrm{m}$, the repeatable precision is $13.49 \mu \mathrm{m}$, and the position deviation is 551.86 $\mu \mathrm{m}$.

\section{Analysis of the Conversion from Angle Error to Positioning Error}

The angle error of the robotic arm measured in this study was converted from the angle $\theta$ to the positioning error in the $x$ - and $y$-directions using the robotic kinematic formula. Figure 14 shows the errors of the J1 axis in the $x$-direction before and after the calibration, and Fig. 15 shows the errors of the $\mathrm{J} 1$ axis in the $y$-direction before and after the calibration. Figure 16

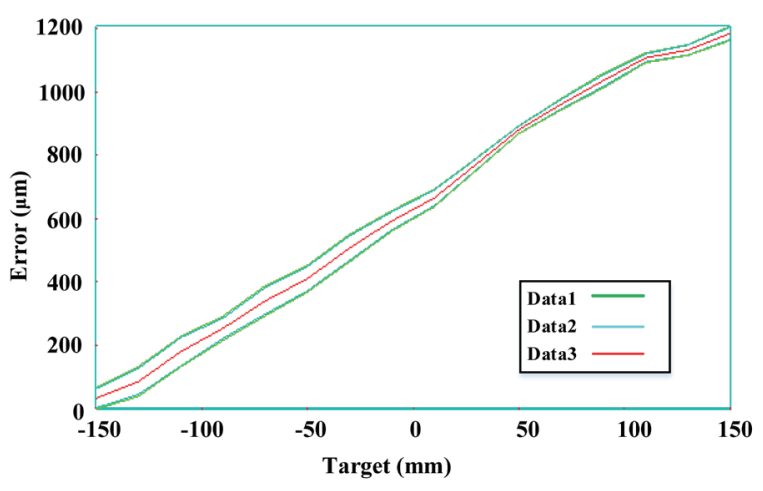

Fig. 12. (Color online) $y$-axis linear length measurements of joint axes $\mathrm{J} 1$ and $\mathrm{J} 2$ before calibration.

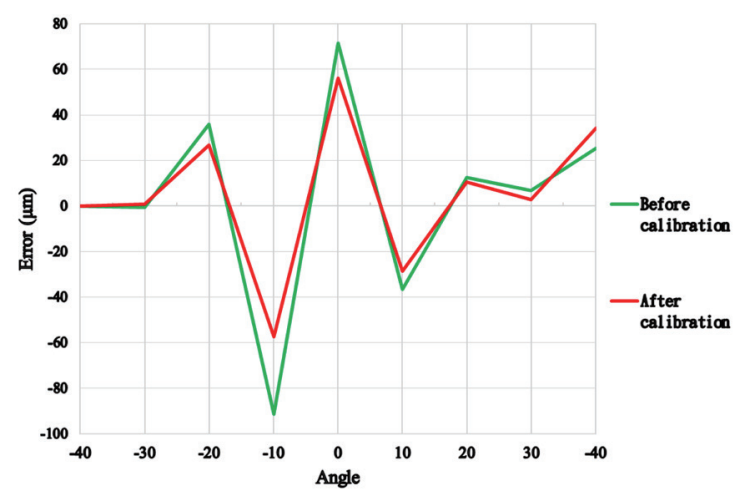

Fig. 14. (Color online) Positioning error variation of the $\mathrm{J} 1$ axis in the $x$-direction.

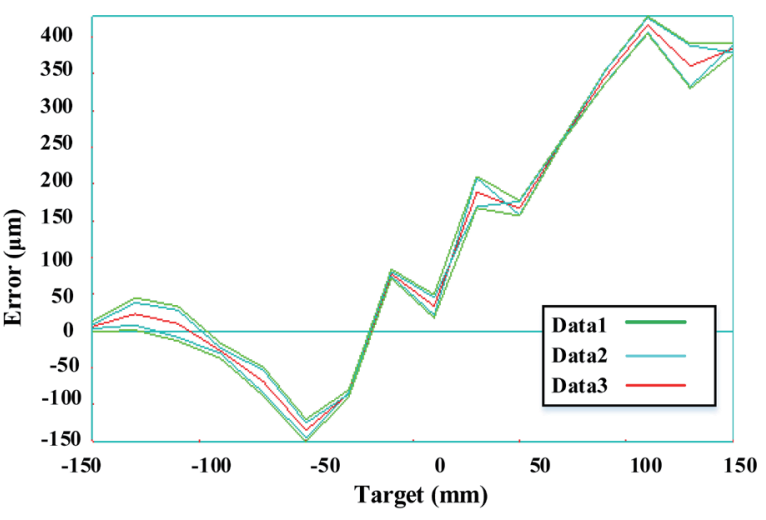

Fig. 13. (Color online) $y$-axis linear length measurements of joint axes $\mathrm{J} 1$ and $\mathrm{J} 2$ after calibration.

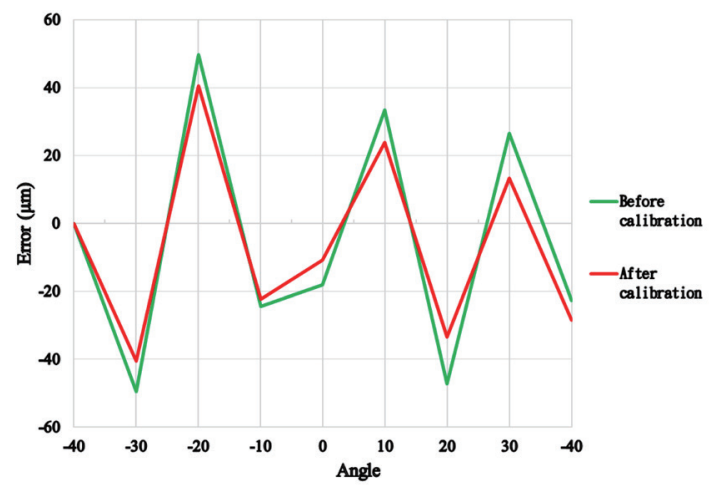

Fig. 15. (Color online) Positioning error variation of the $\mathrm{J} 1$ axis in the $y$-direction. 


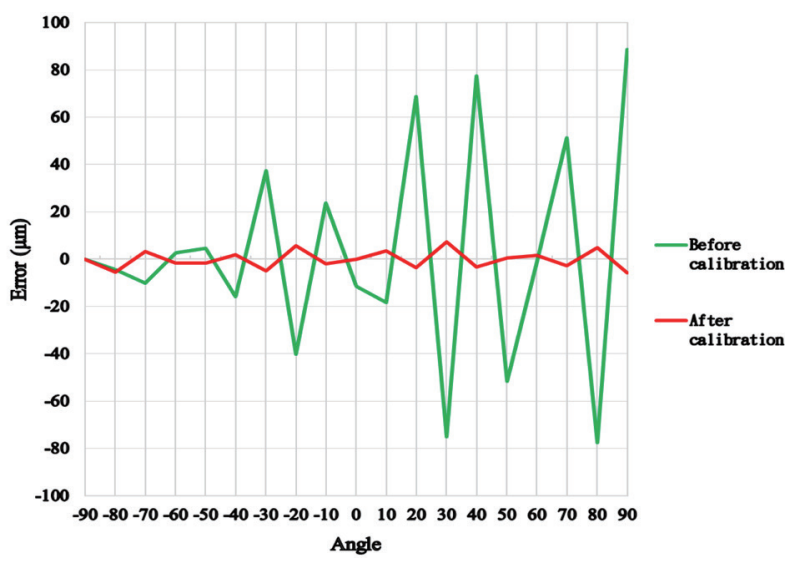

Fig. 16. (Color online) Positioning error variation of the $\mathrm{J} 2$ axis in the $x$-direction.

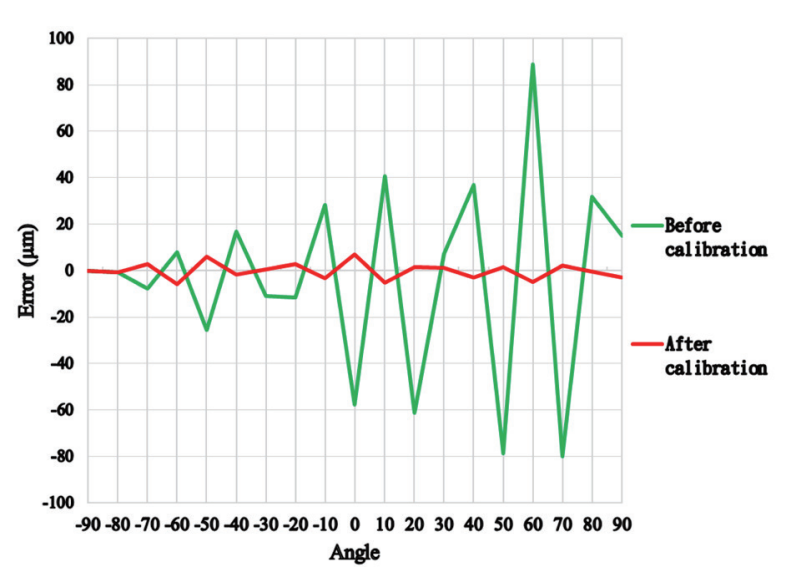

Fig. 17. (Color online) Positioning error variation of the $\mathrm{J} 2$ axis in the $y$-direction.

shows the error variation of the $\mathrm{J} 2$ axis in the $x$-direction, and Fig. 17 shows the error variation of the $\mathrm{J} 2$ axis in the $y$-direction.

The calibration device developed in this study can measure the errors of two joint axes of the SCARA. It focuses on system miniaturization and fast measurement to considerably lower the system cost and substantially reduce the volume. The device has excellent accuracy and can be installed quickly in the robotic arm to shorten the measurement time substantially. The angle errors of the $\mathrm{J} 1$ and $\mathrm{J} 2$ axes are compensated with reference to the measurement results. The positioning accuracy of the $\mathrm{J} 1$ axis is increased to $26.5 \operatorname{arcsec}$ and the repeatable precision is improved to $8.1 \mathrm{arcsec}$. The positioning accuracy of the $\mathrm{J} 2$ axis is increased to $7.3 \mathrm{arcsec}$ and the repeatable precision is improved to 2.3 arcsec. These results reveal that the proposed system can effectively measure the axial angle error of the SCARA, and the angular positioning accuracy can be increased after the compensation of each joint axis of the SCARA.

\section{Conclusions}

A novel joint angle measurement system was proposed in this study and applied to SCARA robotic positioning error compensation. The structure of the measurement system is very simple; it includes a laser measurement device, a reflective mirror, and a high-precision index table. Since the measurement system is based on a high precision index table, the measurable range is wide. The features of the laser measurement device are its high resolution ( 0.5 arcsec), high repeatability ( 1 arcsec), and high accuracy $( \pm 3$ arcsec). However, the measurable range is small (within $\pm 0.1^{\circ}$ ). The measurement system merges the advantages of the index table and the laser measurement device, so that the measurement system is highly suitable for SCARA measurement. The experimental results showed that the angular positioning error deviation for the joints can be increased considerably after calibration. The proposed system is currently a prototype. The measurement signal is processed by a computer. For commercial application, our next task is to process the measurement signal using a microprocessor or a system on chip (SoC) instead. 


\section{Acknowledgments}

This work was supported by the Ministry of Science and Technology under Grant Nos. MOST 106-2218-E-005-014 and MOST 107-2634-F-005-001.

\section{References}

1 N. W. Simpson: Sci. Technol. ProQuest Dissertations Publishing, Canada (2004).

L. Giovani, M. Chiara, and T. James: J. Rob. Syst. 13 (1996) 445.

O. Alberto, L. Giovanni, and A. Riccardo: J. Rob. Syst. 17 (2000) 291.

4 Y. Liu, X. Ning, G. Zhang, X. L. Chen, H. C. Zhang, J. Michael, and T. A. Fuhlbrigge: Int. Conf. Intelligent Robots and Systems (2009) 715.

5 C. S. Gatla, R. Lumia, J. Wood, and G. Starr: J. Rob. Syst. 6 (2000) 291.

6 D. Biqiang, X. Ning, and N. Erick: Int. J. Smart Sens. Intell. Syst. 4 (2012) 987.

7 D. J. Bennett, J. M. Hollerbach, and D. Geiger: Int. J. Rob. Res. 10 (1991) 550.

8 G. R. Tang and H. F. Lan: Int. J. Rob. Autom. 21 (1993) 5.

9 D. W. Osborn and W. S. Newman: Proc. Int. Conf. Robotics and Automation 2 (1993) 160.

10 H. Zhuang, S. H. Motaghedi, and Z. S. Roth: Int. Conf. Robtics and Automation 14 (1999) 805.

11 J. Yin and Y. Gao: Energy Procedia 14 (2012) 977.

12 Y. Tong, Y. Huang, L. Yang, and C. Li: Mechanism and Machine Science Proc. ASIAN MMS 2016 \& CCMMS 2016 (2016) 317.

13 H. W. Lee, T. P. Chiu, and C. H. Liu: Sens. Mater. 28 (2016) 1053.

14 H. W. Lee, J. R. Chen, B. L. Ho, and C. C. Wang: Sens. Mater. 29 (2017) 1471.

15 G. Y. Zhuang, H. W. Lee, and C. H. Liu: Rev. Sci. Instrum. 85 (2014) 105004.

16 T. Yandayana, S. A Akgöza, and H. Haitjemab: J. Int. Soc. Precis. Eng. 26 (2002) 412.

17 H. W. Lee and C. H. Liu: Smart Sci. 4 (2016) 134.

\section{About the Authors}

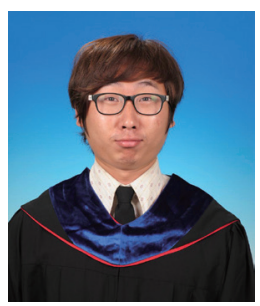

Chun-Kai Lan received his B.S. and M.S. degrees from National Chin-Yi University of Technology, Taiwan, in 2009 and 2012, respectively. Since 2013, he has been pursuing a Ph.D. degree at National Chung Hsing University. His research interests are in the simulation of mold flow in engineering, robotics, solid mechanics, and numerical analysis.

(d102061011@mail.nchu.edu.tw)

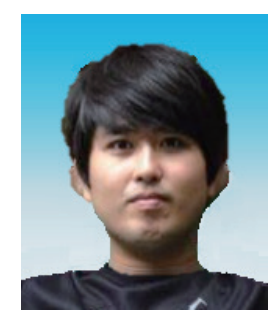

Chung-Yu Wu received his B.S. degree from National Kaohsiung First University of Science and Technology, Taiwan, and his M.S. degree from National Chung Hsing University, Taiwan. His research interests are in robotics, solid mechanics, and mechanism design.

(wuchunyu10507@gmail.com) 


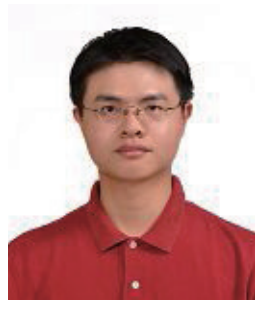

Chih-Wen Chang received his B.S. degree from National Taiwan University of Science and Technology, Taiwan, in 2002 and his M.S. and Ph.D. degrees from National Taiwan Ocean University, Taiwan, in 2004 and 2009, respectively. From 2009 to 2016, he was an associate researcher at the National Center for High-Performance Computing, Taiwan. Since 2017, he has been a researcher at National Chung Hsing University. His research interests are in the simulation of energy engineering, AI algorithms, robotics, solid mechanics, numerical analysis, fluid mechanics, thermodynamics, dynamic systems, plasticity, friction dynamics, inverse problems, ordinary differential equations, partial differential equations, Lie algebra, Lie-group numerical methods, animation effects, and $\mathrm{CO}_{2}$ geologic storage.

(chihwen.chang8@gmail.com)

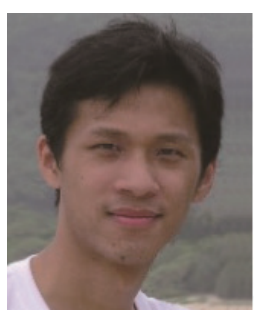

Hau-Wei Lee received his Ph.D. degree from the Institute of Aeronautics and Astronautics, National Cheng-Kung University, Taiwan, Republic of China, in 2010. From 2014 to 2017, he was a researcher at the Center for Measurement Standards, International Technology Research Institute, Taiwan. He is now a researcher at the Department of Mechanical Engineering, National Chung Hsing University, Taiwan. His main research fields are precision instrument design, dimension metrology, calibration of machine tools, and optomechatronic integration engineering.

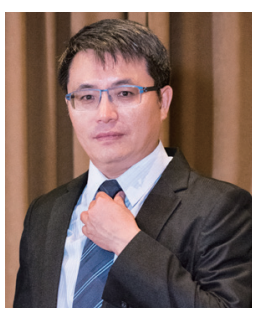

Chien-Hung Liu received his Ph.D. degree in mechanical engineering from National Cheng Kung University (NCKU) in 2000. His current research interests focus on high-precision instrument and control system design, laser engineering, optical measurement, smart sensors, and optical devices. Dr. Liu taught at National Formosa University (NFU) from 2004 to 2011 and was promoted to professor in the Department of Electro-Optical Engineering in 2010. He now teaches in the Department of Mechanical Engineering at National Chung Hsing University (NCHU), Taiwan. During his years in the mechanical engineering field, he has published over 80 papers in SCI journals. Moreover, he holds more than 70 patents. (carus@dragon.nchu.edu.tw) 